\title{
SEQUENTIAL RESPONDING IN ACCORDANCE WITH TEMPORAL RELATIONAL CUES: A COMPARISON OF BEFORE AND AFTER
}

\author{
John M. Hyland \\ Dublin Business School \\ Denis P. O’Hora \\ National University of Ireland, Galway \\ Julian C. Leslie and Sinéad Smyth \\ University of Ulster, Coleraine
}

\begin{abstract}
The current study investigated the relative effects of Before and After relational cues on temporal order judgments. In Experiment 1, participants $(\mathrm{N}=20)$ were exposed to a 5-phase temporal relational responding task. Participants observed a sequence of 2 familiar shapes and then completed either a Before or an After statement to describe the sequence. Response speeds were significantly faster for Before statements than for After statements. Experiment $2(\mathrm{~N}=24)$ extended Experiment 1 , using abstract rather than familiar stimuli, and replicated the findings. The current data extend previous research, which employed temporal relational responding tasks as a measure of cognitive abilities such as intelligence, by focusing on differences in speed between responding in the presence of relational cues used in such tasks. The differences in response speeds observed between Before and After cues suggest that more work is needed to understand the specific processes that underpin such responding.

Key words: before, after, relational responding, temporal judgment
\end{abstract}

The ability to understand and sequence events may be attributed in part to the relations we derive between events and objects. These relations range from simple physical discriminations, such as size (e.g., A is larger than B, B is smaller than A), to more complex ones, such as analogy (e.g., A1B1 is to C1D1 as A2B2 is to C2D2). Early work on transposition posited the idea that people often respond to stimuli based on the stimuli's relative properties (e.g., choosing the brighter of two stimuli) as opposed to their absolute properties (e.g., responding to stimuli of a certain brightness; e.g., Herbert \& Krantz, 1965; Reese, 1968). More recently, researchers (e.g., Sidman, 1994) have argued that equivalence relations may provide a behavioral account of development of language

Part of the current research was presented at the Annual Convention of the Association for Behavior Analysis, Chicago, May 2008.

The current research formed part of John M. Hyland's doctoral thesis, completed at the University of Ulster while he was funded by the Vice Chancellor's research scholarship from 2006.

Correspondence concerning this article should be addressed to John M. Hyland, School of Psychology, Dublin Business School, 13-14 Aungier St., Dublin 2, Ireland. E-mail: john.hyland@dbs.ie 


\section{DBS Library}

\section{Copyright}

The copyright of this article is the property of the Psychological Record.

\section{Usage}

You are free:

to Share - to copy, distribute, display, and perform the work.

Under the following conditions:

Attribution - you must give the original author credit.

Non-commercial - you may not use this work for commercial purposes.

No derivative works - you may not alter, transform, or build upon this work.

This copy has been supplied on the understanding that it is copyright material and that no quotation from this article may be published without proper acknowledgement. 
and similar cognitive processes. Relational frame theory (RFT) in turn extended equivalence research, positing that language-able humans can learn to respond in accordance with derived relations other than Same and Different (Hayes, Barnes-Holmes, \& Roche, 2001).

Most experimental analyses of relational responding employ response accuracy or acquisition as dependent variables. Recently, however, a number of studies have used response time measures to explore differences in responding in accordance with derived relations (Bush, Sidman, \& de Rose, 1989; Fields, Adams, Verhave, \& Newman, 1990; Fields \& Verhave, 1987; O’Hora, Roche, Barnes-Holmes, \& Smeets, 2002; Spencer \& Chase, 1996; Steele \& Hayes, 1991). Steele and Hayes (1991) investigated three types of relational responding: Same, Different, and Opposite. Response latencies were calculated to measure differences in responding to the combinatorially entailed Same relations that were the result of combining either Same (e.g., If A is the same as B and B is the same as $\mathrm{C}$, then $\mathrm{A}$ is the same as $\mathrm{C}$ ) or Opposite relations (e.g., If $\mathrm{A}$ is the opposite of $\mathrm{B}$ and $\mathrm{B}$ is the opposite of $\mathrm{C}$, then $\mathrm{A}$ is the same as $\mathrm{C}$ ). Participants responded more quickly in accordance with combinatorially entailed relations based on trained Same relations (i.e., shorter response latencies) than with those based on Opposite relations. The authors suggested that the longer reaction times for Opposite-based relational responding were due to deriving a different relation from that trained. A similar study by O’Hora et al. (2002) compared latencies of mutually entailed relational responses (e.g., if A is the same as B, then it can be derived that B is the same as A). The research focused on the relations More Than, Less Than, Same, and Opposite. In More Than and Less Than relations, the mutually entailed relation is different than that trained (e.g., If A is more than B, then B is less than A, whereas if A is the same as B, then B is the same as A).

The Before/After relation allows humans to verbally sequence behaviors in a particular order. Many daily routines, such as getting dressed and making breakfast, require us to arrange subroutines in particular sequences (e.g., boiling a kettle requires filling the kettle with water before heating it). One key difference between Before and After statements relates to the coordination of the verbal descriptions with the event (see Clark, 1971). In this line of research, the idea of coordination referred to descriptions of events with Before cues and how these descriptions coordinate with the temporal order in which the events occur (e.g., "Fill the kettle before you heat it"). In contrast, although descriptions that employ After relational cues denote the same actions, they describe the event in a reverse order (e.g., "Heat the kettle after you have filled it"). An alternative interpretation of children's responding in the presence of Before and After cues was put forward by Amidon and Carey (1972). They asserted that the difficulty arises from the presence of a subordinate clause. The study consisted of children responding to instructions containing both Before and After cues with subordinate clauses (e.g., "Move the red plane after you move the blue plane") and instructions with no such clauses (e.g., "Move the red plane first; move the blue plane last”). The latter instruction still maintains the temporal order of the events but does not contain a subordinate clause. Amidon and Carey's views contrasted with Clark's semantic hierarchical account, as the relations First/Last should still exhibit the same features of time, simultaneity, and prior that Before/After exhibit. It could therefore be suggested that children understand temporal order quite well, just not when Before and After occasion subordinate clauses.

There have been few studies of temporal relational responding within the relational responding paradigm. O’Hora, Peláez, and Barnes-Holmes (2005) investigated the correlations between Before/After relational responding and performance on verbal subtests of the Wechsler Adult Intelligence Scale-Third Edition (WAIS-III; Wechsler, 1997). Participants were tested on a behavioral model of instructional control for Before and After relational responding and were subsequently required to complete several subtests of the WAIS-III. It was found that successful completion of the complex relational task was correlated with higher scoring on the Vocabulary subtest of the Verbal 
Comprehension index of the WAIS-III. More specifically, moderately strong correlations were observed between temporal relational task scores and correct responses on the Vocabulary subtest. O’Hora et al. (2008) further investigated the relationship between temporal relational responding and intelligence. Specifically, the study examined whether performance on relational responding tasks would predict performance on the four indices of the WAIS-III. Based on RFT predictions, it was expected that high performance on relational responding tasks would predict similar performance on the Verbal Comprehension and Perceptual Organization indices. These expectations were developed on the premise that relational ability underpins many verbally based cognitive abilities. It was also expected that responding scores would not predict scores on the other two indices, Working Memory and Processing Speed, because the procedure did not require more than minimal input from memory. The predictions were supported through subsequent analysis, with participants who successfully completed the temporal relational task scoring high on the Verbal Comprehension and Perceptual Organization indices but not on the Working Memory or Processing Speed indices. These findings support RFT claims that such relational ability underlies a number of cognitive abilities.

A recent study by O’Toole and Barnes-Holmes (2009) extended O’Hora et al.'s (2005) findings on relational responding and intelligence scores by using response time as an indicator of fluency in relational responding tasks. The relational responding task used by O’Toole and Barnes-Holmes was the Implicit Relational Assessment Procedure (IRAP; see Barnes-Holmes et al., 2006), during which participants were required to respond to or agree with statements that were either consistent (e.g., spring before summer) or inconsistent (e.g., spring after summer). The IRAP works on the premise that responding to consistent trials will be faster than responding to inconsistent trials. The difference in response latency was found to correlate negatively with measures of intelligence. Specifically, they found that the smaller the differential between consistent and inconsistent trials, the higher the score on the measure of intellectual flexibility. This led the authors to suggest that their procedure may provide a measure of intellectual flexibility. Though interesting, O'Toole and Barnes-Holmes's procedure differed substantially from that of O'Hora et al. O'Hora et al. compared responding to Before and After relations, while O'Toole and BarnesHolmes examined the difference between responding to consistent (or well-established) relations and inconsistent relations, regardless of whether the relations were Before or After. They found that those who scored higher on the intelligence test exhibited greater relational flexibility, the ability to respond in conflict with globally well-established verbal relations.

The findings of Steele and Hayes (1991) and O’Hora et al. (2002) have important implications, as they suggested that responding to such relations (i.e., Before/After instructions) takes more time. Both O’Hora et al. and Steele and Hayes used response latency measurements - the latter explored response differences between mutually entailed relations containing Same and Opposite relational cues, and the former extended this by also looking at More Than and Less Than. These studies demonstrated the importance of measuring response latencies for such responding, as differences in the relative time taken for responding to these relations were found. In line with the Clark (1971) notion of Before/After coordination, if a participant is presented with an A...B sequence, a corresponding Before description would coordinate with it (e.g., if A...B, then A before $\mathrm{B})$, but if the corresponding description consisted of an After cue, a relation must be derived in order for the participant to choose the stimuli in the correct sequence (e.g., if A...B, then B after A). Responding to After cues may take more time to construct, so it is expected that the response speed for After instructions would be slower than for Before instructions.

The current study attempted to extend understanding of Before/After relational responding by employing both accuracy and response time measures to investigate differences between the specific Before and After relational cues. Verbally describing sequences 
of events involves using the relational cues of either Before or After (e.g., A1 before B1, B1 after A1). Describing sequences using Before requires a repetition of the observed sequence, but describing sequences using After requires a reversal of the observed sequence. Based on the findings of Clark (1971) and the research on coordination and combinatorially and mutually entailed relations by Steele and Hayes (1991) and O'Hora et al. (2002), this reversal is likely to require more time to provide the description. Experiment 1 measured the speed of order judgments that included either Before or After relational cues. It was expected that the response speed of After judgments would be slower than that of Before judgments.

\section{Experiment 1}

\section{Method}

Participants. Twenty undergraduate and postgraduate students (5 male and 15 female) at the University of Ulster participated in this study. Written informed consent was obtained from all participants. All participants were experimentally naive. No financial reward or research credit was offered in exchange for taking part. All participants were over 18 years of age, spoke English as their first language, and did not have a language disorder. Participants were randomly assigned to one of the two groups, Before-After (BA) or After-Before (AB), before the experiment began.

Materials and apparatus. Participants were seated in $2 \mathrm{~m} \times 2 \mathrm{~m}$ experimental cubicles in the psychology laboratory. There were two types of stimuli used in the current study (see Figure 1): those used in observed sequences and those used in the response array. The stimuli used in the observed sequences consisted of four familiar shapes (circle, square, triangle, and cross) and were $4 \mathrm{~cm}^{2}$ in area. The stimuli presented in arrays after the observed sequence cleared from the screen were the same four geometric shapes but with an area of $1.5 \mathrm{~cm}^{2}$. Size was set using the IrfanView program (Skiljan, 2007). The English words before and after were used as relational cues in the subsequent response statements. These were black, capitalized, and set in bold, 24-point Times New Roman font. The present study was programmed by the first and second authors in Visual Basic 6.0. A Fujitsu Siemens Scenic 300 personal computer and accompanying 15-inch CRT monitor were used for stimulus presentation. Responses were made in each trial by clicking with a standard computer mouse.
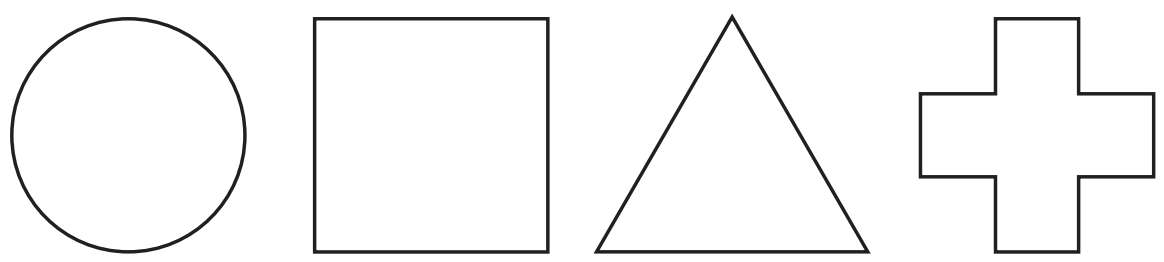

Figure 1. An illustration of the geometric stimuli used in the study. In the observed sequences, stimuli were set at $4 \mathrm{~cm}^{2}$ in area, and the corresponding stimuli presented in the subsequent arrays were $1.5 \mathrm{~cm}^{2}$ in area.

Procedure. Participants were exposed to a five-phase sequential responding procedure (see Figure 2). The five phases are referred to as Before Training, Before Probes, After Training, After Probes, and Mixed Probes. There were 12 trial types in the Before Training phase. Each trial type comprised two geometric shapes in a particular sequential order (e.g., circle...square; cross...triangle). All four geometric shapes were randomly assigned to a particular position in the sequence, with all permutations of shape and order presented once in a quasirandom order (see Figure 3). Thus, participants were exposed to each of the 12 trial types once in a quasirandom order. 


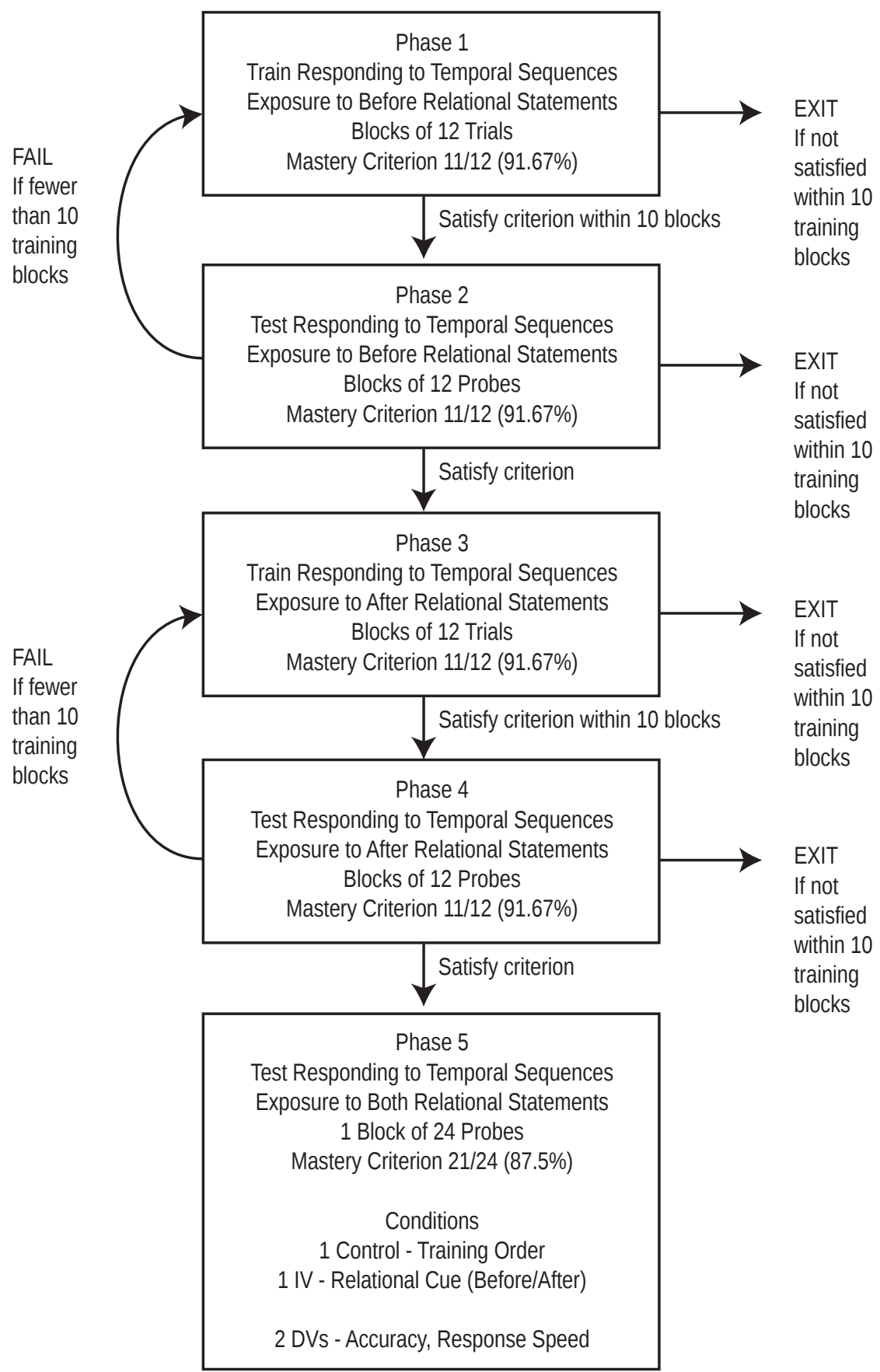

Figure 2. An experimental outline of participant progression in the BA group. Participants were initially exposed to training and probe phases employing Before cues. In the next phase, the After Training phase, After relational statements were presented and trained. This phase was followed by the After Probes phase. In the AB group, participants began with the After Training phase, and this was followed by the After Probes phase. These phases were followed by the Before Training and the Before Probes phases, in that order. Both groups finished the experiment with the Mixed Probes phase. 

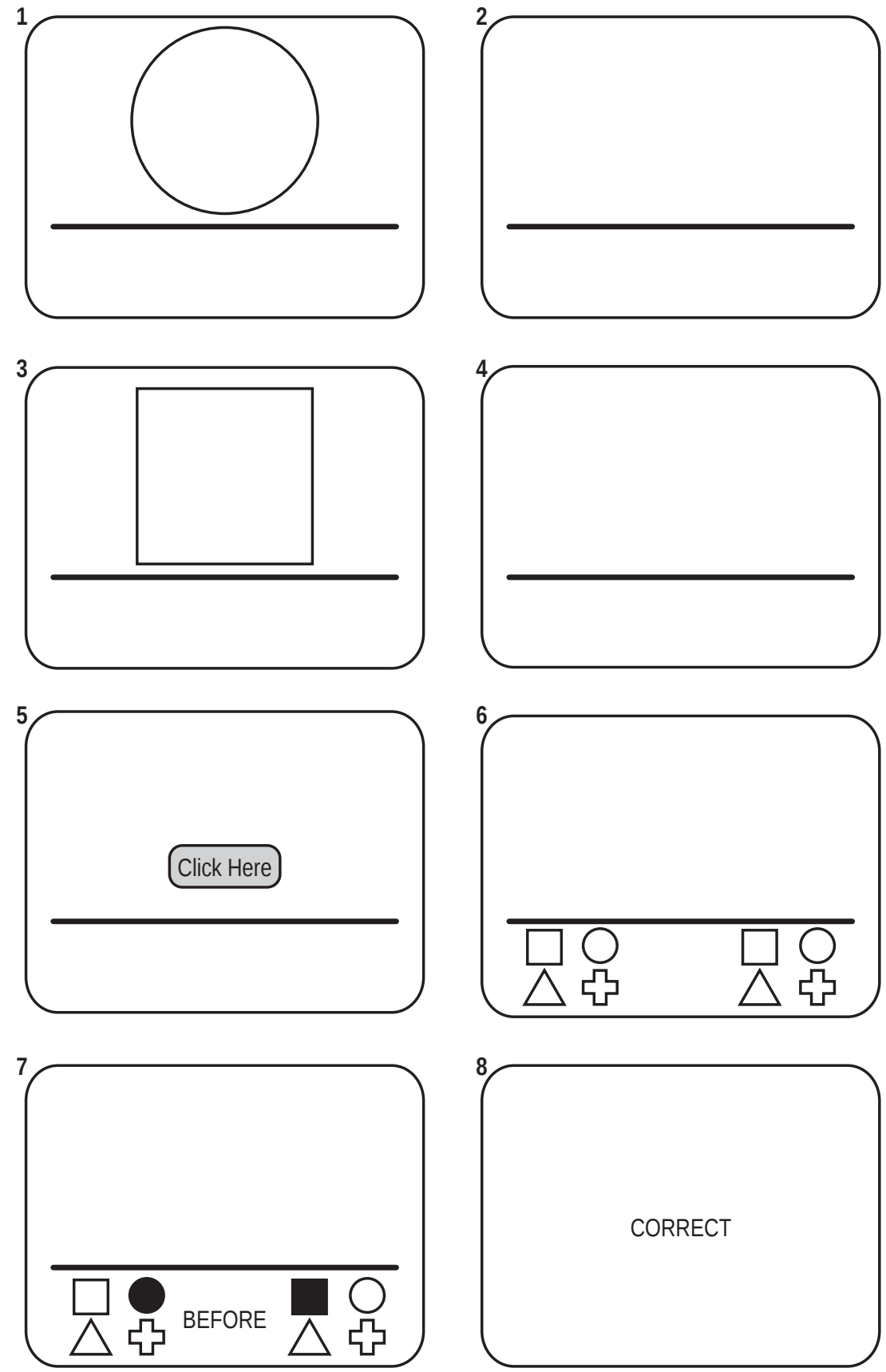

Figure 3. An outline of the successive screens seen by a participant during a Before Training trial (e.g., in Phase 1). The panel numbers indicate the order in which participants progressed through the trial. Screens 1 to 4 outline the presentation of each stimulus and the ITI after each presentation. Screens 5 and 6 illustrate the presentation of the message box, with the button click revealing a screen with two arrays of four shapes below a black line on both the bottom left and bottom right hand side of the screen. Screen 7 highlights the correct stimuli chosen, with the final screen highlighting the feedback presented. 
Instructions for the Before Training phase were presented as follows:

This phase of the experiment requires you to concentrate on a sequence of 2 shapes at the top of the screen. One shape will appear for a brief moment and then disappear. Another shape will then appear in the same position for an equal amount of time. A second after the second shape disappears, a message box will appear underneath. You are required to click on the message box, which will reveal several shapes on both the bottom left and bottom right hand sides of the screen beneath a black line. You are required to choose one shape from either side. In between both sets of shapes there is a word. This word will in some way relate both shapes you choose.

In the experiment, one of two words will appear in different trials. You will try to learn how this word relates the shapes during each training session. You must respond from left to right in each trial. In this first stage, you will be provided with feedback. When you're ready, click on the button below.

In a typical Before Training phase trial, participants observed a sequence of two shapes, randomly chosen from the pool of four. The first shape appeared in the center of a white screen for $1,000 \mathrm{~ms}$. The screen cleared for a 1,000-ms interstimulus interval (ISI), which consisted of a blank white screen. The ISI appeared at several points between stimulus-stimulus and stimulus-response presentations. The second shape appeared after the ISI for 1,000 ms and was replaced by another 1,000-ms ISI. After the second ISI, a grey message box containing the words "Click Here" appeared in the center of the screen (see Figure 3). After the participant clicked the message box, the screen cleared and a black line appeared, marking out the bottom quarter of the screen. Beneath the black line, two arrays of four shapes were positioned at the bottom of the screen, one on the left hand side and one on the right. The position of the message box also controlled for mouse cursor position, with the mouse icon equidistant from each shape in the array, positioned at the center of the word BEFORE (see Figure 3). The word BEFORE was positioned at the bottom center of the screen, separating the arrays. Participants were required to click on one of the four shapes in the bottom left of the screen and then on one of the four shapes in the bottom right (see Figure 3). Correct responding on trials reflected the order in which the shapes in the sequence had just appeared. In a typical example, the sequence of circle followed by square was presented. In the presence of the Before relational cue, choosing the circle in the left array followed by the square in the right array was correct, as the observed sequence was circle before square.

Feedback was provided in the form of the messages "Correct" in green writing, to reinforce correct responses, and "Wrong" in red writing after incorrect responses. Participants were required to choose a shape from the left array followed by a shape from the right array, so responding from right to left resulted in negative corrective feedback. Once feedback had been given, an intertrial interval (ITI) consisting of a blank white screen appeared for 1,000 ms before the next trial was presented in exactly the same manner. This continued until all 12 trials had been presented in a quasirandom order. Participants were required to demonstrate responding at a criterion level of 11 out of 12 trials in order to move on to the Before Probes phase. Failure to reach criterion resulted in retraining on the Before Training phase. A maximum of 10 exposures to the Before Probes phase was provided. Failure to demonstrate responding at mastery level within this number of exposures resulted in the termination of the study. See Figure 2 for an illustration of experimental progression.

The following instructions were presented to participants at the beginning of the Before Probes phase: 
This phase of the experiment requires you to respond in the same way for each trial as Phase 1 . Remember to respond from left to right in each trial. The only difference in this phase is that no feedback will be presented after each trial.

Good luck! When you're ready, click on the button below.

The Before Probes phase was identical to the Before Training phase, except that no feedback was presented for responses. Participants demonstrating mastery criterion on Before Probes progressed to the After Training phase, and those who failed Before Probes were exposed to retraining on the Before Training phase. Upon completion of the Before Probes phase, the following After Training phase instructions appeared:

This phase of the experiment requires you to respond in the same way for each trial as Phase 1. Feedback will be presented after each trial in this stage. Remember to take your time and understand the sequence of shapes and how they are related, depending on the word in between.

Good luck! When you're ready, click on the button below.

The After Training phase was identical to the Before Training phase except that the relational cue After, instead of Before, was presented between the arrays. All trial types outlined in the Before Training phase were the same as for the After Training phase. In the example outlined above, in the presence of the After cue, choosing the square followed by the circle would be correct, as the statement would correctly describe the observed sequence Square after Circle. The mastery criterion was identical to the previous two phases, and those who were successful progressed to the After Probes phase. Failure to respond at mastery level resulted in retraining on the After Training phase.

After Probes instructions were presented to participants as follows:

This phase of the experiment requires you to respond to shapes in a certain sequence as in the previous phase. No feedback will be presented after each trial in this phase.

Good luck! When you're ready, click on the button below.

The After Probes phase was identical to the After Training phase apart from the absence of feedback. Those who achieved mastery criterion proceeded to the Mixed Probes phase. Failure resulted in retraining on the After Training phase.

Mixed Probes instructions were presented as follows:

This phase of the experiment requires you to respond to the sequence of shapes as in previous stages. No feedback will be presented after each trial. Remember to take your time and understand each trial, and to respond from left to right in all trials.

Good luck! When you're ready, click on the button below.

The Mixed Probes phase was a 24-trial phase, in which all trial types were presented twice, once in the presence of the Before relational cue and once in the presence of the After cue. Feedback was not given in this phase, and mastery criterion was set as 21 correct responses out of 24 possible trials. Successful completion of the Mixed Probes phase resulted in the conclusion of the experiment. Failure to demonstrate mastery criterion level resulted in further exposure to the Mixed Probes phase.

In the BA group, participants were exposed to the phases in the following order: Before Training, Before Probes, After Training, After Probes, and Mixed Probes. In the AB group, the order of phases was as follows: After Training, After Probes, Before Training, Before Probes, and Mixed Probes. This controlled for any learned order and facilitation effects of the relational cue. 


\section{Results and Discussion}

The raw data consisted of accuracy and reaction time scores for each participant for each of the five experimental phases. All 20 participants completed the experiment within nine blocks. There were 10 participants in the BA group and 10 participants in the AB group.

Accuracy data. Accuracy data are presented in Tables 1 and 2. Eight of the 20 participants reached mastery criterion on all five phases at the first attempt (four in the BA group and four in the AB group). An additional eight participants completed the Before Training phase on the first attempt (four in the BA group and four in the AB group), with three of the remaining four requiring one additional exposure (one in the BA group and two in the $\mathrm{AB}$ group) and one participant (in the BA group) requiring three additional training blocks before reaching criterion.

Table 1

Number of Trials Completed by BA Group Participants in Each Phase Before Criterion Was Met

\begin{tabular}{cccccc}
\hline Participant & Before Training & Before Probes & After Training & After Probes & Mixed Probes \\
\hline P1 & $12 / 12$ & $12 / 12$ & $11 / 12$ & $12 / 12$ & $23 / 24$ \\
P2 & $12 / 12$ & $12 / 12$ & $11 / 12$ & $12 / 12$ & $24 / 24$ \\
P3 & $12 / 12$ & $12 / 12$ & $21 / 24$ & $12 / 12$ & $24 / 24$ \\
P4 & $11 / 12$ & $12 / 12$ & $12 / 12$ & $11 / 12$ & $24 / 24$ \\
P5 & $12 / 12$ & $11 / 12$ & $29 / 36$ & $12 / 12$ & $22 / 24$ \\
P6 & $11 / 12$ & $12 / 12$ & $32 / 36$ & $12 / 12$ & $24 / 24$ \\
P7 & $12 / 12$ & $12 / 12$ & $11 / 12$ & $12 / 12$ & $24 / 24$ \\
P8 & $21 / 24$ & $12 / 12$ & $15 / 24$ & $12 / 12$ & $53 / 72^{\mathrm{a}}$ \\
P9 & $12 / 12$ & $12 / 12$ & $22 / 24$ & $12 / 12$ & $42 / 48^{\mathrm{a}}$ \\
P10 & $40 / 48$ & $12 / 12$ & $12 / 12$ & $12 / 12$ & $23 / 24$ \\
\hline
\end{tabular}

Note. The first number in each cell refers to the number of correct trials, and the second number refers to the total number of trails completed. Blocks for Before Training, Before Probes, After Training, and After Probes all consisted of 12 trials per block. Mixed Probes blocks consisted of 24 trials. Eight participants passed the Mixed Probes phase on the first attempt.

aparticipant required additional exposure on the Mixed Probes phase before reaching criterion.

Table 2

Number of Trials Completed by AB Group Participants in Each Phase Before Criterion Was Met

\begin{tabular}{cccccc}
\hline Participant & After Training & After Probes & Before Training & Before Probes & Mixed Probes \\
\hline P1 & $11 / 12$ & $12 / 12$ & $11 / 12$ & $12 / 12$ & $24 / 24$ \\
P2 & $11 / 12$ & $12 / 12$ & $12 / 12$ & $12 / 12$ & $24 / 24$ \\
P3 & $26 / 24$ & $11 / 12$ & $22 / 24$ & $11 / 12$ & $21 / 24$ \\
P4 & $12 / 12$ & $12 / 12$ & $11 / 12$ & $12 / 12$ & $24 / 24$ \\
P5 & $21 / 24$ & $12 / 12$ & $11 / 12$ & $12 / 12$ & $24 / 24$ \\
P6 & $22 / 24$ & $12 / 12$ & $22 / 24$ & $11 / 12$ & $24 / 24$ \\
P7 & $21 / 24$ & $11 / 12$ & $12 / 12$ & $12 / 12$ & $21 / 24$ \\
P8 & $21 / 24$ & $11 / 12$ & $12 / 12$ & $12 / 12$ & $23 / 24$ \\
P9 & $11 / 12$ & $12 / 12$ & $12 / 12$ & $12 / 12$ & $21 / 24$ \\
P10 & $22 / 24$ & $12 / 12$ & $11 / 12$ & $12 / 12$ & $24 / 24$ \\
\hline
\end{tabular}

Note. The first number in each cell refers to the number of correct trials, and the second number refers to the total number of trails completed. Blocks for Before Training, Before Probes, After Training, and After Probes all consisted of 12 trials per block. Mixed Probes blocks consisted of 24 trials. All participants passed the Mixed Probes phase on the first attempt. 
All participants, including those who required additional exposure to the Before Training phase, passed the Before Probes on the first attempt. Apart from the eight participants who passed all phases on the first attempt, one additional participant (from the BA group) passed After Training on the first attempt, and an additional nine participants reached criterion on the second exposure (three in the BA group and six in the AB group). Two more participants (from the BA group) required three exposures before reaching criterion. All participants passed the After Probes phase on the first attempt. Eighteen participants completed the Mixed Probes phase on the first exposure (eight in the BA group and 10 in the $\mathrm{AB}$ group), with one participant (from the BA group) requiring one additional exposure and the remaining participant (from the BA group) requiring two exposures to reach criterion. See Tables 1 and 2 for participant progression in both control groups.

Response speed data. There are two measures of speed calculated. First, reaction time data were analyzed in terms of response speed, which was taken as the reciprocal of the response latency in seconds (1 divided by the latency in seconds; see Hall, Sekuler, \& Cushman, 1969, for an overview on calculating response speed). The Mixed Probes phase response speeds were used in the analysis between groups (see Figure 4 for an illustration of the findings). In the Mixed Probes phase, the mean response speed in the BA condition was faster for Before probes $(M=0.67, S D=0.13)$ than for After probes $(M=0.56$, $S D=0.15$ ). The AB group also yielded similar differences between means of Before $(M=0.63, S D=0.09)$ and After $(M=0.54, S D=0.09)$ probes. The mean response speed also exhibited faster average responding rates for Before probes $(M=0.65, S D=0.11)$ than for After probes $(M=0.55, S D=0.12)$ in both groups. There were no notable differences between Before probes in the BA group $(M=0.67)$ and Before probes in the AB group $(M=0.63)$. Similarly, no notable differences were found between mean response speeds on After responding in the BA group $(M=0.56)$ and responding in the AB group $(M=0.54)$. Average response speeds across both $\mathrm{BA}$ and $\mathrm{AB}$ for Before probes $(M=0.65)$ and After probes $(M=0.55)$ corresponded to reaction times of $1.54 \mathrm{~s}$ and $1.82 \mathrm{~s}$, respectively.

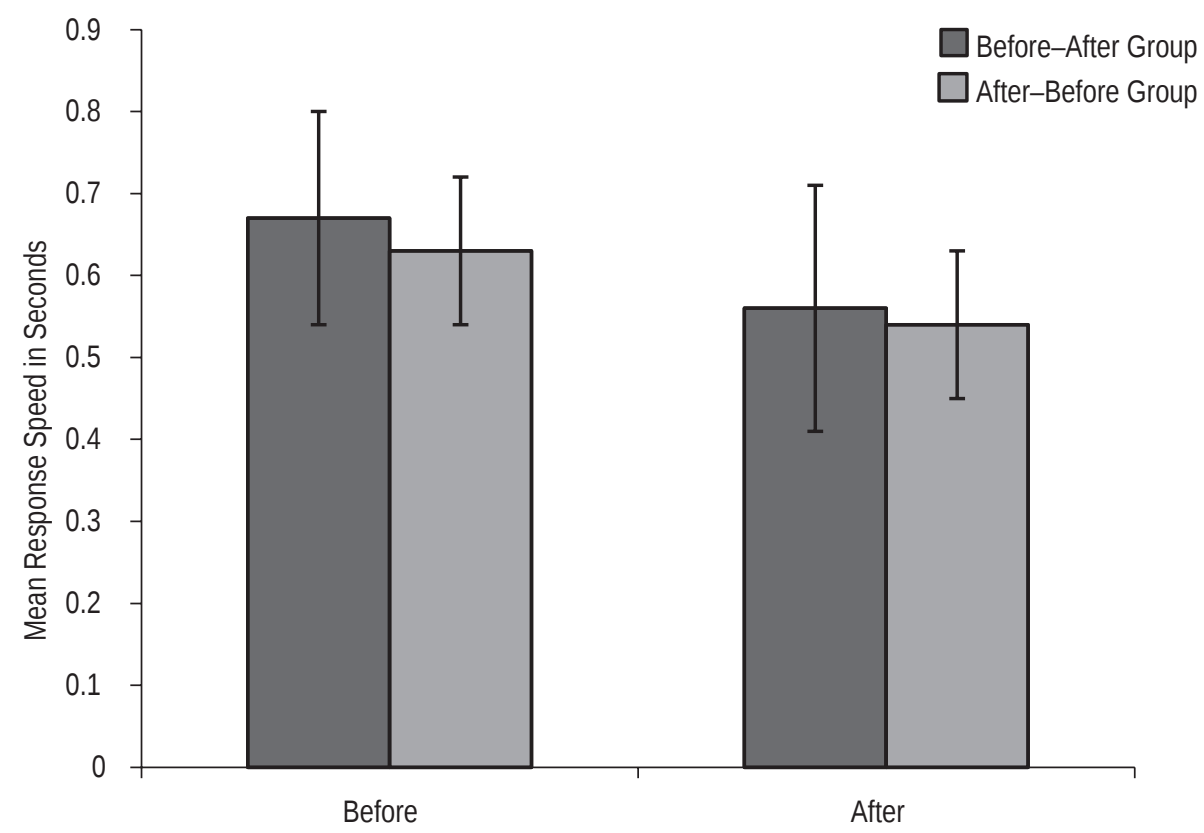

Trial Type

Figure 4. Descriptive statistics for the BA and AB groups. Means and standard deviations for both groups were calculated. Notable differences in mean response speeds were found, with Before responding faster than After responding in both groups. No differences were found between the means across groups. 
A two-way 2 (response type: Before/After) $\times 2$ (training order: BA/AB) mixed analysis of variance (ANOVA) examined the differences in response speed due to these factors. Mean response speed for Before Probes was significantly faster than for After Probes, $F(1,16)=28.6, p<0.001$, partial $\eta^{2}=0.641$. No significant difference was found between training order groups, $F(1,16)=0.225, p=0.642$, partial $\eta^{2}=0.014$, and there was no interaction between response type and group, $F(1,16)=0.331, p=0.573$, partial $\eta^{2}=0.02$.

The second reaction time measure analyzed was interresponse times (IRTs), which corresponded to the latency between choosing the first and second stimulus from the response array. When choosing the first and second stimulus from each of the comparison arrays, it is possible IRTs would be significantly faster in Before responding than in After because of associative conditioning. In other words, the second response made in the sequence may be primed by its association with the first comparison response. Response speeds were calculated using the procedure outlined previously. Mean IRT speeds for Before $(M=1.04)$ and After responding $(M=1.02)$ were calculated across both groups. A second two-way 2 (response type: Before/After) $\times 2$ (response order: BA/AB) mixed ANOVA was conducted to investigate whether there were differences in IRTs when participants described sequences using either Before or After cues. No differences were found in the IRTs for both response types, $F(1,16)=0.38, p=0.546$. There were also no differences in responding across control groups, $F(1,16)=0.013, p=0.91$, and no significant interaction, $F(1,16)=0.03, p=0.862$.

Experiment 1 investigated how humans verbally respond to stimulus sequences and how employing particular temporal cues (e.g., Before or After) can affect time taken to describe such sequences. Participants responded significantly faster when asked to describe sequences with Before cues than with After cues, irrespective of the order in which responding to these cues was trained.

One issue with Experiment 1 concerned the use of Visual Basic (Microsoft, 1998) to measure response speeds. Previous research (André, Ghio, Cavé, \& Teston, 2003; Asaad \& Eskandar, 2008) identified potential technical problems with some types of stimulus presentation programs that may lead to the inaccurate measuring of response speeds. One feature they identified as affecting precise measurement was the unplanned delays caused by parallel background processes and applications. The program used in the current study was developed in Microsoft Visual Basic Version 6.0, which is a high-level language that operates on a non-real time operating system, Microsoft Windows. Consequently, we were unable to control for background processes that compete for computational power with stimulus presentation programs and may have induced delays in the response measures. One way to control for this potential problem is by programming stimulus presentations in low-level languages (e.g., $\mathrm{C}++$ ) that allow the user to control and inhibit background processes. Alternatively, software packages such as Presentation or E-Prime provide easy-to-use development environments that set controls on background processes and stimulus presentation hardware in order to maximize temporal resolution. It may be argued that the results obtained in Experiment 1 could have been subject to temporal measurement problems as outlined above.

Experiment 2 sought to control for such problems. Nevertheless, in the current experiment, Before and After trials were presented randomly during the critical test sessions, so it is unlikely that there was a directional bias caused by the lower temporal resolution. In fact, the likely effect of lower temporal resolution would have been to increase overall variability and make it less likely to obtain a significant difference between conditions.

Employing familiar stimuli in Experiment 1 may have made it more likely that participants would verbalize the relationship between the sequence stimuli as they observed them. That is, as the participants watched a circle appear followed by a square, they may have verbalized the rule "circle before square." This is especially likely because they were aware that they would be asked to judge the order of these stimuli. It is therefore possible 
that the higher response speeds were due to the correspondence between the required judgment and a rule verbalized while watching the sequence. Holth and Arntzen (1998) reviewed numerous studies that investigated the effects of stimulus familiarity on equivalence class formation and found that participants found it easier to derive relations between familiar stimuli than between unfamiliar stimuli. Similarly, Arntzen (2004) found that equivalence class formation occurred more readily when participants were exposed to familiar stimuli earlier in equivalence training. According to Arntzen, this may be due to familiar nameable stimuli facilitating partitioning of novel stimuli into equivalence classes, as such stimuli are already grouped based on their verbal labels. Indeed, much research has been conducted in the past 20 years identifying the effects of exposure to arbitrary and nonarbitrary stimuli (e.g., O’Connor, Rafferty, Barnes-Holmes, \& Barnes-Holmes, 2009).

In addition, in the literature related to working memory (Baddeley \& Hitch, 1974), Hulme, Maughan, and Brown (1991) explored the effects of long-term memory on shortterm memory span, the largest number of verbal stimuli that can be recalled in the learned serial order. Hulme et al. compared words and nonwords to demonstrate long-term effects on recall and found a significantly higher recall of words than of nonwords. As the procedure in Experiment 1 used nonarbitrary stimuli, it could be argued that respondents benefited from this when establishing stimulus relations. It may be posited, based on the above studies, that differences could be found between relations containing arbitrary and nonarbitrary stimuli.

Based on these issues, novel, unfamiliar stimuli were employed for Experiment 2. The experimental program used for Experiment 2 was written in E-Prime Version 2.0, in order to enhance the temporal resolution of the response measures. Furthermore, based on the assertions of Holth and Arntzen (1998) and Arntzen (2004), novel arbitrary stimuli were used for each experimental phase, so that responding on Phase 2 and subsequent phases could not be attributed to a history of responding to the experimental stimuli.

\section{Experiment 2}

\section{Method}

Participants. Twenty-four undergraduate and postgraduate students (9 male and 15 female) from the University of Ulster participated in this study. The same recruitment criteria outlined in Experiment 1 were also used for Experiment 2. Participants were once again randomly assigned to one of two groups, the BA group or the AB group. Written informed consent was obtained from all participants.

Materials and apparatus. The setting and computer used were exactly the same as for Experiment 1; however, the software program and stimuli differed. The program was written by the first and second authors in the E-Prime Version 2.0 software application suite. This experiment differed from Experiment 1 in that arbitrary, rather than nameable, stimuli were employed (see Figure 5). Furthermore, novel stimulus sets were employed for each phase. As in Experiment 1, stimuli presented in observed sequences were $4 \mathrm{~cm}^{2}$ in area, and stimuli presented in the descriptive arrays were $1.5 \mathrm{~cm}^{2}$ in area. Stimulus sizes were controlled for using Irfanview (Skiljan, 2007). The English words before and after were used as relational cues in the present study. These cues were capitalized and set in bold, black, 24-point Times New Roman font. The fixation point was black in color and was positioned in the center of the screen. During training, feedback in the form of the messages "Correct" and "Wrong" were presented in green and red, respectively, 24-point, bold Times New Roman font. 
Training
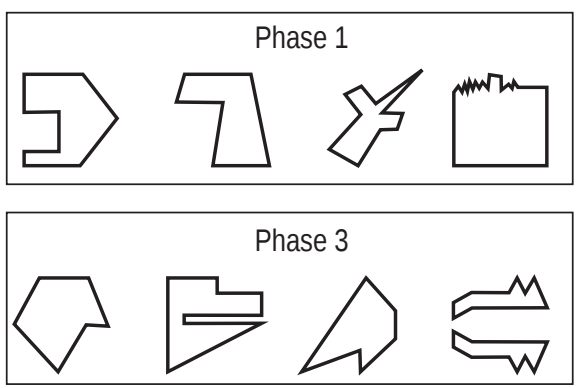

Probes

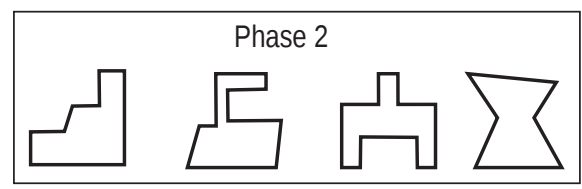

Phase 4

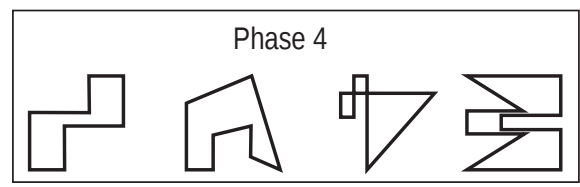

Phase 5

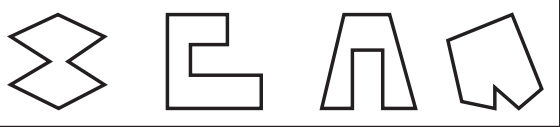

Figure 5. The arbitrary stimuli used in Experiment 2. Stimuli in each section were used in each of the five phases in the study.

Procedure. Experiment 2 was procedurally similar to Experiment 1 . The following section only describes the methodological differences between Experiments 1 and 2. In the current experiment, each phase had an exclusive pool of four arbitrary shapes. As described in Experiment 1, participants in the BA group were exposed to the phase order Before Training, Before Probes, After Training, After Probes, and Mixed Probes, and participants in the AB group were given the phase order of After Training, After Probes, Before Training, Before Probes, and Mixed Probes.

An outline of a typical trial can be seen in Figure 6. As in Experiment 1, there were 12 trial types in the first four phases. In the sequences at the beginning of each trial, two shapes appeared in a randomly assigned order. All permutations of two shape sequences were presented once in a quasirandom order. Participants were required to concentrate on the sequence of two shapes in the center of a screen. The first stimulus appeared for $1,000 \mathrm{~ms}$, and then the screen cleared for an ISI of 1,000 ms. A second stimulus then appeared for the same duration, and the screen subsequently cleared for an ISI. After the second ISI, a fixation point appeared in the same position as the shapes in the center of the screen. The fixation point persisted for 1,000 ms.

The fixation point was replaced by an array of four shapes to the left center of the screen and an array of four shapes to the right center of the screen. The word BEFORE appeared in the center of both arrays. Each of the four shapes appeared once in both arrays, and the position of the shapes within the array remained the same for all 12 trials in the phase. The relational cue and the mouse icon appeared in the same position that the fixation point had been in before it was replaced. This controlled for the position of the mouse icon, which was equidistant from the comparison arrays. Participants were required to choose one shape from the left side and then a shape from the right. As in Experiment 1, mastery criterion was set at 11 correct responses out of 12 trials for the first four phases and 21 out of 24 trials for the Mixed Probes phase. Participants had only one exposure to the Mixed Probes phase and therefore had only one attempt to reach criterion level before the study ended. This was another difference between Experiments 1 and 2; this change was made to ensure responding on the Mixed Probes phase was not a result of learning from repeated exposure. 

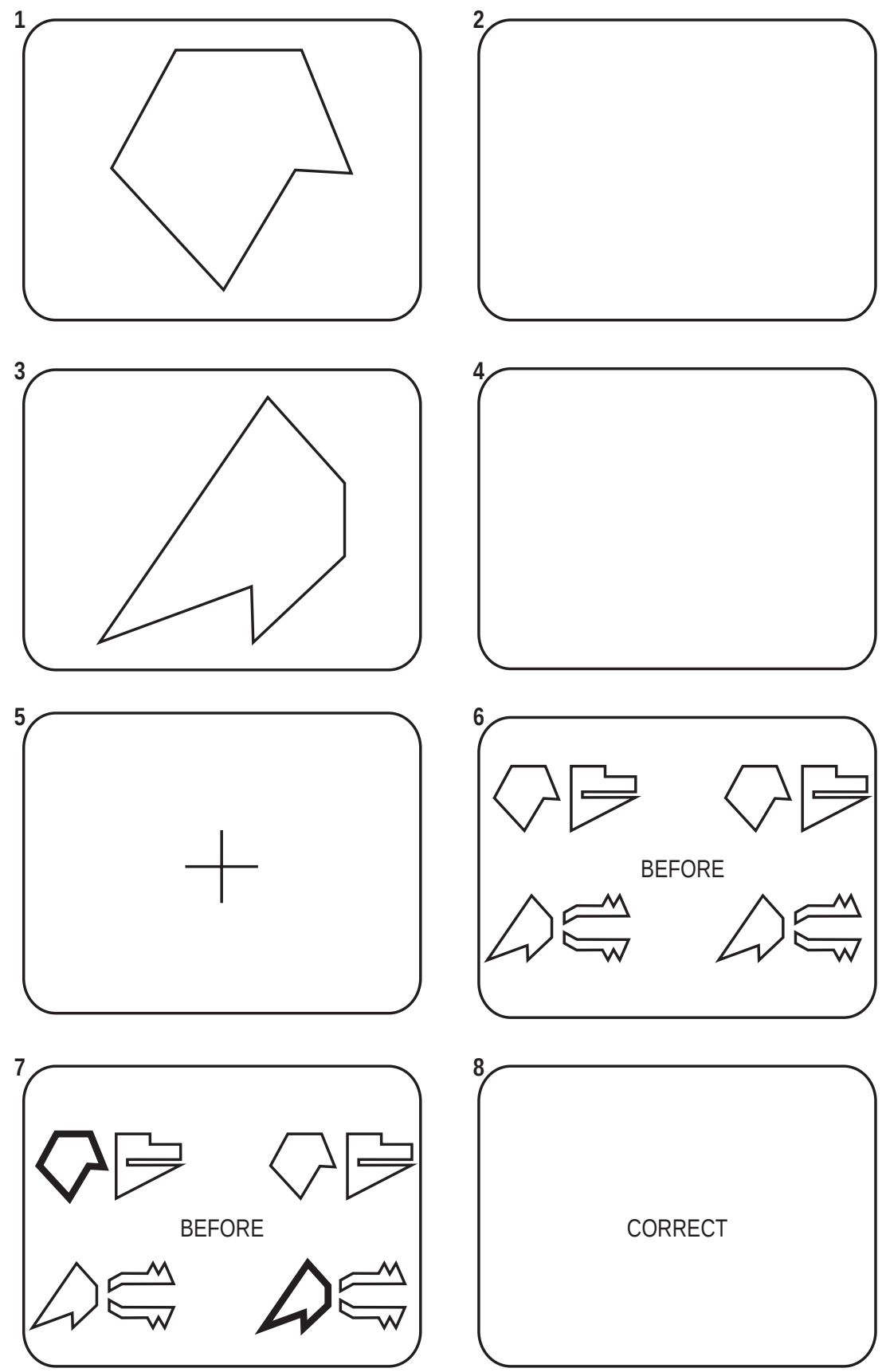

Figure 6. An example of a Before trial in Experiment 2. In the second-to-last screen, the stimuli outlined in bold show the observed sequence, and choosing these would result in the message "Correct" appearing on the screen. 


\section{Results}

As in Experiment 1, the raw data consisted of accuracy and reaction time scores for each participant for each of the five experimental phases. All 24 participants completed the experiment. Twelve participants were assigned to the BA group and 12 participants to the $\mathrm{AB}$ group. As in Experiment 1, analysis was performed on the accuracy data. Acquisition and responding was very high across both groups, similar to results in Experiment 1. Reaction time data were further analyzed in terms of response speed, and analyses were performed to explore differences in the relational cue and across both BA and $\mathrm{AB}$ groups.

Accuracy data. All 24 participants completed the experiment. Participants in the BA group completed the experiment phases in the order of Before Training, Before Probes, After Training, After Probes, and Mixed Probes, with participants in the AB group completing the phases in the order of After Training, After Probes, Before Training, Before Probes, and Mixed Probes.

Data from one participant in the BA group was omitted because the average response speed in the Mixed Probes phase fell outside 1.5 standard deviations from the mean. The low average speed was not simply caused by individual trial outliers; consistently low speed responding across all trials contributed to this average as well. This was observed in prior training trials, and training did not seem to increase response speed. Two participants reached mastery criterion on all five phases on the first attempt (two in the BA group). These data are presented in Table 3. An additional 13 participants completed the Before Training phase on the first attempt (three in the BA group and 10 in the AB group), with 13 additional participants requiring one additional exposure to reach criterion level (four in the $\mathrm{BA}$ group and two in the $\mathrm{AB}$ group). Three participants required two additional exposures to reach criterion level on the Before Training phase (three in the BA group). All 24 participants passed the Before Probes phase on the first attempt.

In addition to the two participants who passed all phases on the first attempt, an additional nine participants passed the After Training phase on the first exposure (three in the BA group). An additional 12 participants passed After Training with one additional training block (three in the BA group and nine in the AB group), with three participants (from the BA group) requiring two additional exposures. Two participants required three additional exposures before reaching criterion level on After Training (one in the BA group and one in the $\mathrm{AB}$ group), and one participant (from the $\mathrm{AB}$ group) required four additional exposures. All participants, except for two (from the AB group), passed After Probes on the first exposure, and 22 of the remaining 24 participants reached mastery criterion on Mixed Probes in the first attempt. Two participants (from the AB group) did not reach criterion on Mixed Probes. The results of these participants were omitted from reaction time analysis. See Tables 3 and 4 for participant progression.

Response speed data. The Mixed Probes phase response speeds were used in the analysis between conditions (see Figure 7 for an illustration of the findings). Speed was calculated as the inverse of latency responses. The mean response speed in the BA group was notably faster for Before probes $(M=0.54, S D=0.1)$ than for After probes $(M=0.48$, $S D=0.13$ ). The $\mathrm{AB}$ condition yielded similar differences between means of Before probes $(M=0.5, S D=0.12)$ and After probes $(M=0.46, S D=0.16)$. The mean response speed for Before probes in both groups $(M=0.52, S D=0.13)$ also exhibited faster average responding rates than for After probes $(M=0.47, S D=0.14)$. There were also small differences between Before probes in the BA group $(M=0.54)$ and Before probes in the AB condition $(M=0.5)$. Similarly, a small difference was observed between mean response speeds on After probes responding in the BA group $(M=0.48)$ and responding in the $\mathrm{AB}$ condition $(M=0.46)$. Overall response speeds for Before probes and After probes corresponded to reaction times of $1.92 \mathrm{~s}$ and $2.12 \mathrm{~s}$, respectively. 
Table 3

Number of Trials Completed by BA Group Participants in Each Phase Before Criterion Was Met

\begin{tabular}{cccccc}
\hline Participant & Before Training & Before Probes & After Training & After Probes & Mixed Probes \\
\hline P1 & $12 / 12$ & $12 / 12$ & $21 / 36$ & $12 / 12$ & $24 / 24$ \\
P2 & $11 / 12$ & $12 / 12$ & $12 / 12$ & $12 / 12$ & $24 / 24$ \\
P3 & $22 / 24$ & $12 / 12$ & $12 / 12$ & $12 / 12$ & $24 / 24$ \\
P4 & $31 / 36$ & $11 / 12$ & $30 / 36$ & $11 / 12$ & $21 / 24$ \\
P5 & $21 / 24$ & $12 / 12$ & $12 / 12$ & $12 / 12$ & $24 / 24$ \\
P6 & $21 / 24$ & $12 / 12$ & $19 / 24$ & $12 / 12$ & $22 / 24$ \\
P7 & $29 / 36$ & $11 / 12$ & $11 / 12$ & $11 / 12$ & $22 / 24$ \\
P8 & $22 / 24$ & $12 / 12$ & $19 / 24$ & $12 / 12$ & $22 / 24$ \\
P9 & $12 / 12$ & $12 / 12$ & $12 / 12$ & $12 / 12$ & $24 / 24$ \\
P10 & $12 / 12$ & $12 / 12$ & $20 / 24$ & $11 / 12$ & $23 / 24$ \\
P11 & $11 / 12$ & $12 / 12$ & $12 / 48$ & $11 / 12$ & $23 / 24$ \\
P12 & $27 / 36$ & $11 / 12$ & $17 / 36$ & $12 / 12$ & $24 / 24$ \\
\hline
\end{tabular}

Note. The first figure in each cell refers to the number of correct trials, and the second number refers to the total number of trails completed. Blocks for Before Training, Before Probes, After Training, and After Probes all consisted of 12 trials per block. Mixed Probes blocks consisted of 24 trials.

Table 4

Number of Trials Completed by AB Group Participants in Each Phase Before Criterion Was Met

\begin{tabular}{cccccc}
\hline Participant & After Training & After Probes & Before Training & Before Probes & Mixed Probes \\
\hline P1 & $22 / 24$ & $12 / 12$ & $11 / 12$ & $12 / 12$ & $24 / 24$ \\
P2 & $25 / 60$ & $12 / 12$ & $12 / 12$ & $12 / 12$ & $23 / 24$ \\
P3 & $16 / 12$ & $12 / 12$ & $21 / 24$ & $12 / 12$ & $22 / 24$ \\
P4 & $22 / 24$ & $12 / 12$ & $11 / 12$ & $12 / 12$ & $24 / 24$ \\
P5 & $21 / 48$ & $12 / 12$ & $12 / 12$ & $12 / 12$ & $24 / 24$ \\
P6 & $22 / 24$ & $21 / 24^{\mathrm{a}}$ & $12 / 12$ & $12 / 12$ & $24 / 24$ \\
P7 & $48 / 60$ & $22 / 24^{\mathrm{a}}$ & $11 / 12$ & $12 / 12$ & $24 / 24$ \\
P8 & $19 / 24$ & $12 / 12$ & $21 / 24$ & $12 / 12$ & $10 / 24^{\mathrm{b}}$ \\
P9 & $19 / 24$ & $12 / 12$ & $12 / 12$ & $12 / 12$ & $20 / 24^{\mathrm{b}}$ \\
P10 & $22 / 24$ & $12 / 12$ & $11 / 12$ & $12 / 12$ & $24 / 24$ \\
P11 & $22 / 24$ & $11 / 12$ & $12 / 12$ & $12 / 12$ & $23 / 24$ \\
P12 & $21 / 24$ & $11 / 12$ & $12 / 12$ & $12 / 12$ & $24 / 24$ \\
\hline
\end{tabular}

Note. The first number in each cell refers to the number of correct trials, and the second number refers to the total number of trails completed. Blocks for Before Training, Before Probes, After Training, and After Probes all consisted of 12 trials per block. Mixed Probes blocks consisted of 24 trials. Six participants passed all probe phases with one exposure, while two participants failed the Before Probes phase and required additional Phase 1 training.

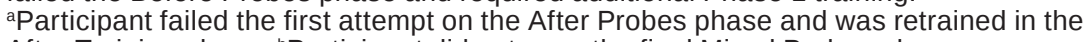
After Training phase. ${ }^{b}$ Participant did not pass the final Mixed Probes phase.

Statistical analyses. A two-way 2 (response type: Before/After) $\times 2$ (response order: $\mathrm{BA} / \mathrm{AB}$ ) mixed ANOVA examined the differences between response type (withinsubjects factor) and response order (between-subjects factor) in terms of speed of responding. A statistically significant difference was found between response type, $F(1,20)=9.04, p=0.007$, partial $\eta^{2}=0.311$. As outlined in the response speed data 
discussed previously, mean response speed for Before probes was notably faster than for After probes. This suggests that participants required additional time to respond to sequences containing the After relational cue. No significant differences were found between response conditions, $F(1,20)<1, p=0.594, \eta^{2}=0.14$, and there was no interaction between response type and group, $F(1,20)<1, p=0.6, \eta^{2}=0.014$.

A second two-way 2 (response type: Before/After) $\times 2$ (response order: BA/AB) mixed ANOVA explored the differences between response type and response order in terms of IRTs. No differences were found in the IRTs for both response types, $F(1,20)<1, p=0.7$. There were also no differences in responding across control groups, $F(1,20)<1, p=0.469$, and no significant interaction, $F(1,20)<1, p=0.92$.

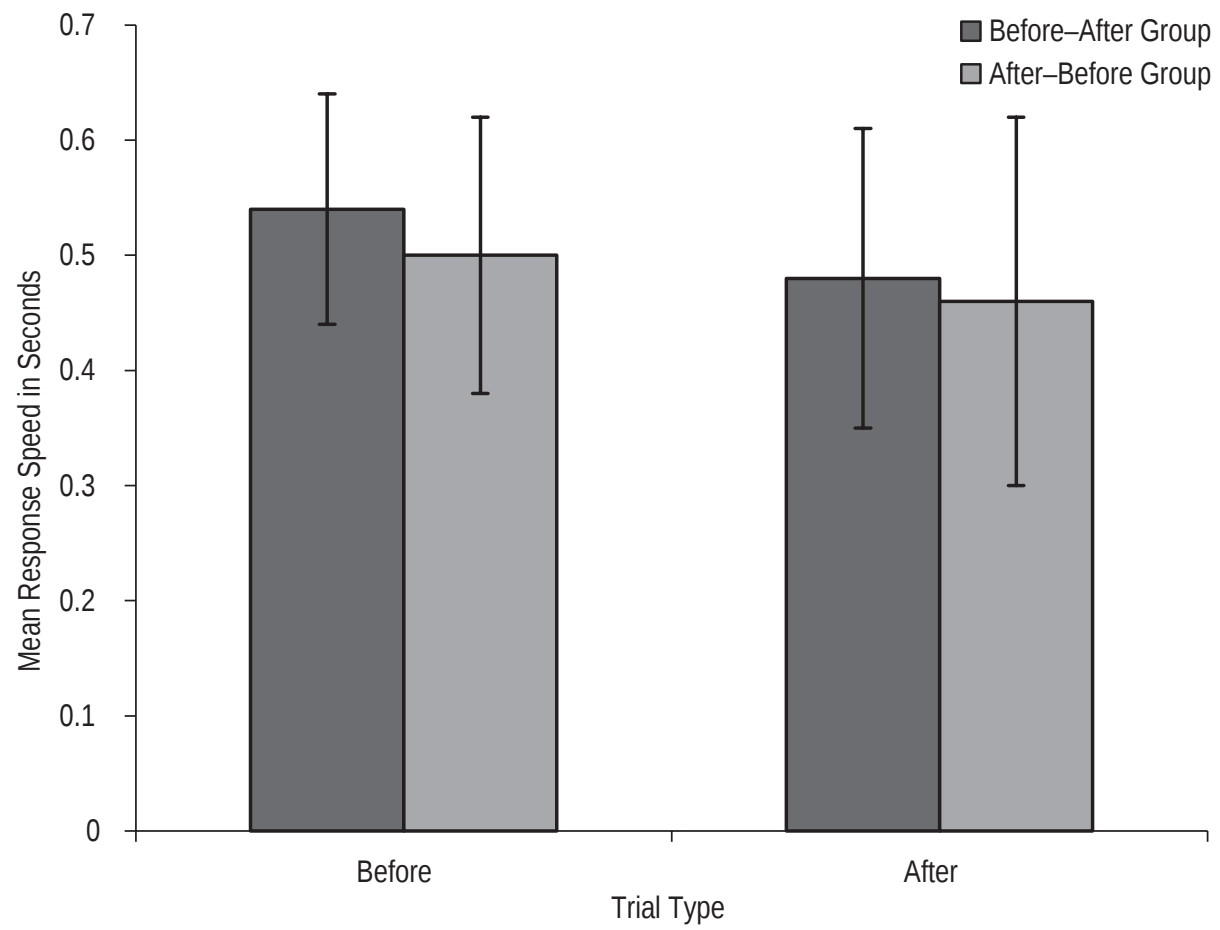

Figure 7. Descriptive statistics for Before and After relational responding. The means and standard deviations were calculated across $B A$ and $A B$ groups. As in Experiment 1, notable differences were observed between response speeds in both Before and After relational responding. There were also notable differences across both $B A$ and $A B$ groups in terms of mean response speed.

In Experiment 2, it was established that response speeds were significantly faster in sequences containing Before relational cues. This experiment replicated the findings in Experiment 1. It was found that responding to sequences containing arbitrary stimuli was not different than responding to sequences containing familiar geometric stimuli. In Experiment 1, stimuli were not replaced throughout phases. Experiment 2 controlled for familiarity of stimuli through replacement of stimuli in each phase.

\section{General Discussion}

The aim of Experiments 1 and 2 was to analyze the role of the temporal relational cues Before and After in describing observed sequences of stimuli. In Experiment 1, the procedure required participants to respond to observed sequences of geometric stimuli by describing such sequences using a relational statement containing either a Before or an 
After cue. Participants demonstrated faster responding when the Before cue was presented, suggesting that temporal coordination between the sequence order and the relational statement affected response speed. No differences were observed between the IRTs for Before and After statements (i.e., response speed between choosing the first and second stimulus in the statement). Experiment 2 replicated these findings while making some methodological refinements. Perhaps most important, these refinements included the use of novel stimuli for testing. This precaution helps support the external validity of the findings by ensuring that generalized relational responding, as opposed to trained responding based on the exact conditional discriminations, was being measured (e.g., Arntzen, 2004; Holth \& Arntzen, 1998; Sidman, 1994).

Some variability can be observed in terms of progression across relational cues. In other words, some participants required additional training to achieve mastery criterion from Phase 2 probes on the first relational cue to Phase 3 training on the second. This was not considered for analysis in these experiments. This was primarily due to participants' requiring more trials to understand the change in coordination of the statement. In earlier pilots of the procedure, it was found that instructing participants to respond from left to right was not enough, as there was still a tendency to respond from right to left. In other words, participants may have assumed that the change in response cue required constructing the statement in the opposite direction, rather than responding consistently to earlier phases and choosing comparison shapes so that the statement coordinated with the new cue. Perhaps more important, counterbalancing controlled for differences in responding in the presence of both cues during the Mixed Probes phase. The Mixed Probes phase was randomized; thus, responding was not affected by expectancy. There is scope for future research to develop a methodology to effectively test progression across cues, as this will further identify differences in such responding.

Some researchers (e.g., Andre et al., 2003) have expressed concern about the use of Microsoft Visual Basic as programming software for developing tightly controlled stimulus displays. Even though we used Visual Basic in Experiment 1, in Experiment 2, we used E-Prime to control for measurement precision, and with this experiment, we replicated the major findings of Experiment 1. The current study provides tentative evidence that, under certain circumstances, Visual Basic programs provide sufficient temporal accuracy to distinguish between responses of interest. The latency differences observed between conditions in the current experiments were in the order of $0.28 \mathrm{~s}$ for Experiment 1 and $0.2 \mathrm{~s}$ for Experiment 2. These were also reliable across participants (In Experiment 1, 18 of 20 participants were faster on Before Probes than on After Probes. In Experiment 2, 18 of 22 participants exhibited faster responding during Before Probes). In such circumstances, it seems that Visual Basic is suitable.

Both Experiments 1 and 2 demonstrate that participants are significantly faster at describing temporal sequences when using a Before relational cue than when using an After relational cue. The methodology used in the current study provides an experimental paradigm with which to analyze such relational responding in adult participants. This was one novel attempt at developing and testing an experimental procedure to investigate the differences between Before and After temporal relations in describing particular sequences. This lends support to previous findings (e.g., O’Hora et al., 2002; Steele \& Hayes, 1991) that responding in accordance with derived combinatorial and mutual entailed relations that are different than those trained takes longer than responding in accordance with derived relations that are the same as those trained.

The present findings replicate those of Clark (1971), based on research on early language development, that Before statements are easier to understand than After statements. Moreover, these data extend Clark's research through the use of adult participants. Clark suggested that Before statements are easier because they preserve the order in which stimuli are observed. Additional research remains to be conducted on temporal relational responding, however, as previous research has found no differences between forward (Before) and reverse (After) temporal statements when less ambiguous terminology, such 
as first and last, is used (Amidon \& Carey, 1972). In addition, it is not clear whether such effects are limited to temporal statements. For instance, further research may investigate whether such reversal effects are observed for statements about relative size (e.g., A ... b ... A is bigger than b or $b$ is smaller than $A$ ).

The response speed differences found in the current study may suggest that a response judgment is made between the presentation of the second stimulus in the observed sequence and the choice of the first stimulus comparison. Donders (1869) analyzed response speeds in relation to additive factors. This was based on the premise that two processes, such as structuring and recognizing a sequence, require more time than the single process of just recognizing the sequence. Similarly, Sternberg (1966) discovered that the magnitude of the comparison digit set increased response time on a digit search task when participants were required to complete a memory recall task. More recent cognitive research (e.g., Kanabus, Szelag, Rojeck, \& Pöppel, 2002) showed that in order for two stimuli to be correctly ordered in a temporal sequence, the ISI between them would need to be at least 20 to $40 \mathrm{~ms}$ (see also Hirsh \& Sherrick, 1961). Similar studies have confirmed this ISI value, suggesting that there may be a process underlying temporal order when responding to audio, visual, and tactile stimulus relations that are independent of the presented stimuli (e.g., Pöppel, 1997).

Previous research into relational responding (e.g., O’Hora et al., 2002; Steele \& Hayes, 1991) has suggested that differences in response latencies may be due to the nature of the relations involved. O'Toole and Barnes-Holmes (2009), however, looked at the difference in responding to familiar stimuli consistently (e.g., spring before summer) versus inconsistently (e.g., spring after summer). The current data could be seen to provide support for both theses. However, it is possible that people have a greater history of responding to Before cues, given that, temporally, this is how stimuli are generally presented in the real world. It is possible, therefore, that the After trials in the current study acted in a manner similar to that of the inconsistent trials employed by O’Toole and Barnes-Holmes. One way to test this proposition would be to train responding to After cues and test to see if the differential in response latency compared to Before cues was subsequently reduced.

Grayson and Wasserman (1979) suggested that short-term memory for sequences of responses may play a role in temporal responding. A particular two-item response sequence, if acting as a functional operant, may be retained in short-term memory until reinforcement or response differentiation takes place (Weisman \& Dodd, 1979). Wasserman, Nelson, and Larew (1980) proposed that animals, such as pigeons, should demonstrate that memory for two-item response differentiation should be available upon time of reinforcement. Pigeons were trained to peck left and right keys in particular sequences upon illumination of the keys, and the intervals between illuminations varied from $0.5 \mathrm{~s}$ to 2 and $4 \mathrm{~s}$. Results indicated that pigeons retained sequence information until reinforcement and that responding was highest in positive trials (i.e., those trials that were positively reinforced). The ITIs between stimuli in the sequence and second stimulus response and subsequent reinforcement were varied and measured across trials. This was to explore whether interitem delay (duration between Stimulus 1 and Stimulus 2) or retention delay (duration between Stimulus 2 and Response 1) would have a greater effect on responding. It was found that increasing the retention level had detrimental effects on responding in positive trials, whereas relatively little effect was found when the interitem duration was varied. Future research could explore the effects of ITI variation on sequential responding and whether response speed and accuracy would decrease as a function of increased ITI.

One methodological limitation of note relates to the instructions used at the beginning of the After Training and Mixed Probes phases of Experiments 1 and 2. Within these instructions, participants were asked to take their time and understand the sequences. The instructions used at the beginning of the Before Training and Before Probes phases did not include such a statement. Considering response speeds were recorded and compared between Before and After trials in the Mixed Probes phases, 
instructing participants to take their time may have resulted in slower response speeds for After trials. Notwithstanding this limitation, the inclusion of this statement should not have affected the validity of the results, considering that response speeds were recorded and compared in the Mixed Probes phase only. In this way, instructions were consistent for responding across both relational statements. It is, however, important that future refinements to this procedure control for such instructional issues. The current data demonstrate a clear differential in responding to two common temporal cues, Before and After. One question that arises from the current study regards the effects of memory on stimulus recall. In contrast to the findings of Hulme et al. (1991), the current Experiment 2 provides support for the notion that the ability to verbally process stimuli has no effect on responding to stimulus sequences. Future research may attempt to extend the current research and investigate the specific differences in terms of memory recall between novel and nonarbitrary stimuli. Varying the ISI between presentations of stimuli may extend our understanding of this control process, particularly in relation to both forward and reverse relational cues. Similarly, varying the duration between second stimulus presentation and comparison stimuli may reveal much about the effects of short-term memory on serial recall. These variations will not only identify important features of order judgments but also explore conflicts between verbal statements and stimulus order and whether this affects one type of relational statement more than another.

\section{References}

AMIDON, A., \& CAREY, P. (1972). Why five-year-olds cannot understand before and after. Journal of Verbal Learning and Verbal Behavior, 11, 417-423.

ANDRÉ, C., GHIO, A., CAVÉ, C., \& TESTON, B. (2003). PERCEVAL: A computer-driven system for experimentation on auditory and visual perception. In M. Sole, D. Recasens, \& J. Romero (Eds.), Proceedings of the XVth International Congress of Phonetic Sciences (pp. 1421-1424). Barcelona, Spain: Casual Productions.

ARNTZEN, E. (2004). Probability of equivalence formation: Familiar stimuli and training sequence. The Psychological Record, 54, 275-291.

ASAAD, F. W., \& ESKANDAR, E. N. (2008). Achieving behavioral control with millisecond resolution in a high-level programming environment. Journal of Neuroscience Methods, 173(2), 235-240.

BADDELEY, A., \& HITCH, G. (1974). Working memory. In G. Bower (Ed.), The psychology of learning and motivation (pp. 47-89). New York, NY: Academic Press.

BARNES-HOLMES, D., BARNES-HOLMES, Y., POWER, P., HAYDEN, E., MILNE, R., \& STEWART, I. (2006). Do you really know what you believe? Developing the Implicit Relational Assessment Procedure (IRAP) as a direct measure of implicit beliefs. The Irish Psychologist, 32, 169-177.

BUSH, K. M., SIDMAN, M., \& DE ROSE, T. (1989). Contextual control of emergent equivalence relations. Journal of the Experimental Analysis of Behavior, 51, 29-45.

CLARK, E. (1971). On the acquisition of the meaning of before and after. Journal of Verbal Learning and Verbal Behavior, 10, 266-275.

DONDERS, F. C. (1869). On the speed of mental processes. Acta Psychologica, 30, 412-431.

FIELDS, L., ADAMS, B. J., VERHAVE, T., \& NEWMAN, S. (1990). The effects of nodality on the formation of equivalence classes. Journal of the Experimental Analysis of Behavior, 53, 345-358.

FIELDS, L., \& VERHAVE, T. (1987). The structure of equivalence classes. Journal of the Experimental Analysis of Behavior, 48, 317-332. 
GRAYSON, R. J., \& WASSERMAN, E. A. (1979). Conditioning of two-response patterns of key pecking in pigeons. Journal of the Experimental Analysis of Behavior, 31, 23-29.

HALL, J. W., SEKULER, R., \& CUSHMAN, W. (1969). Effects of IAR occurrence during learning on response time during subsequent recognition. Journal of Experimental Psychology, 79, 39-42.

HAYES, S. C., BARNES-HOLMES, D., \& ROCHE, B. (2001). Relational frame theory: A post-Skinnerian account of human language and cognition. New York, NY: Plenum.

HIRSH, I. J., \& SHERRICK, C. E. (1961). Perceived order in different sense modalities. Journal of Experimental Psychology, 62, 423-432.

HEBERT, J. A., \& KRANTZ, D. L. (1965). Transposition: A reevaluation. Psychological Bulletin, 63, 244-257.

HOLTH, P., \& ARNTZEN, E. (1998). Stimulus familiarity and the delayed emergence of stimulus equivalence or consistent nonequivalence. The Psychological Record, 48, 81-110.

HULME, C., MAUGHAN, S., \& BROWN, G. D. (1991). Memory for familiar and unfamiliar words: Evidence for a long-term memory contribution to short-term memory span. Journal of Memory and Language, 30, 685-701.

KANABUS, M., SZELAG, E., ROJEK, E., \& PÖPPEL, E. (2002). Temporal order judgement for auditory and visual stimuli. Acta Neurobiologiae Experimentalis, 62, 263-270.

O’ CONNOR, J., RAFFERTY, A., BARNES-HOLMES, D., \& BARNES-HOLMES, Y. (2009). The role of verbal behavior, stimulus nameability and familiarity on the equivalence performances of children. The Psychological Record, 59(10), 53-74.

O’HORA, D., PELÁEZ, M., \& BARNES-HOLMES, D. (2005). Derived relational responding and performance on verbal subtests of the WAIS-III. The Psychological Record, 55, 155-175.

O’ HORA, D., PELÁEZ, M., BARNES-HOLMES, D., RAE, G., ROBINSON, K., \& CHAUDHARY, T. (2008). Temporal relations and intelligence: Correlating relational performance with performance on the WAIS-III. The Psychological Record, 58, 569-584.

O'HORA, D., ROCHE, B., BARNES-HOLMES, D., \& SMEETS, P. M. (2002). Response latencies to multiple derived stimulus relations: Testing two predictions of relational frame theory. The Psychological Record, 52, 51-75.

O’TOOLE, C., \& BARNES-HOLMES, D. (2009). Three chronometric indices of relational responding: The importance of relational flexibility. The Psychological Record, 59(1), 119-132.

PÖPPEL, E. (1997). A hierarchical model of temporal perception. Trends in Cognitive Sciences, 1, 56-61.

REESE, H. W. (1968). The perception of stimulus relations: Discrimination learning and transposition. London, England: Academic Press.

SIDMAN, M. (1994). Equivalence relations and behavior: A research story. Boston, MA: Authors Cooperative.

SKILJAN, I. (2007). IrfanView [Computer software]. Retrieved from http://www.irfanview.com.

SPENCER, T. J., \& CHASE, P. N. (1996). Speed analyses of stimulus equivalence. Journal of the Experimental Analysis of Behavior, 65, 643-659.

STEELE, D. L., \& HAYES, S. C. (1991). Stimulus equivalence and arbitrarily applicable relational responding. Journal of the Experimental Analysis of Behavior, 56, 519-555. 
STERNBERG, S. (1966). High-speed scanning in human memory. Science, 153, 652-654. WASSERMAN, E. A., NELSON, K. R., \& LAREW, M. B. (1980). Memory for sequences of stimuli and responses. Journal of the Experimental Analysis of Behavior, 34, 49-60.

WECHSLER, D. (1997). WAIS-III administration and scoring manual. San Antonio, TX: The Psychological Corporation.

WEISMAN, R. G., \& DODD, P. W. (1979). The study of associations: Methodology and basic phenomena. In A. Dickinson \& R. A. Boakes (Eds.), Mechanisms of learning and motivation: A memorial volume for Jerzy Konorski (pp. 337-363). Hillsdale, NJ: Erlbaum. 